\title{
Effect of passive stretching and jogging on the series elastic muscle stiffness and range of motion of the ankle joint
}

\author{
Peter J McNair, Stephen N Stanley
}

\begin{abstract}
Objective-To determine the effect of stretching and jogging on the series elastic muscle stiffness of the plantar flexors and on the range of dorsiflexion at the ankle joint.
\end{abstract}

Methods-24 healthy subjects participated in this study. Each subject undertook all of the following protocols, in random order: (1) stretching protocol: five 30 s static stretches with 30 s rest between stretches; (2) aerobic jogging protocol: subjects ran on a treadmill for $10 \mathrm{~min}$ at $60 \%$ of their maximum age predicted heart rate; (3) combined protocol: subjects ran first and then stretched. A damped oscillation technique was used to measure the series elastic stiffness of the plantar flexors. Dorsiflexion of the ankle was assessed with a weights and pulley system that moved the ankle joint from a neutral position into dorsiflexion passively. Electromyography was used to monitor the activity of the plantar and dorsiflexors during these procedures. The statistical analysis of these data involved an analysis of covariance

Results-For decreasing series elastic muscle stiffiness running was more effective than stretching $(P<0.05)$. In contrast, the results for range of motion showed that the combination protocol and the stretching only protocol were more effective than the running only protocol $(P<$ 0.05 ) for increasing dorsiflexion range of motion at the ankle.

Conclusions-Both jogging and static stretching exercises appear to be beneficial to individuals participating in sporting activities.

(Br F Sports Med 1996;30:313-318)

of Physiotherapy,

Faculty of Health

Studies, Auckland

Institute of Technology,

Auckland,

New Zealand

P J McNair

$S$ N Stanley

Correspondence to:

Peter J McNair PhD

Neuromuscular Research

Unit, School of

Physiotherapy, Faculty of

Health Studies, Auckland

Institute of Technology,

Private Bag, Auckland,

New Zealand.

Accepted for publication 9 July 1996

Key terms: warm up; stretching; muscle stiffness; joint range of motion

Extensibility in soft tissues is related to the resistance of tissue as it lengthens. The ratio of the change in force to the change in length of the tissue is termed muscle stiffness, ${ }^{1}$ the reciprocol of which is termed compliance. A muscle that is more compliant will lengthen with less resistance. Resistance to stretch will be determined by both mechanical and neural factors. With regard to the former, muscle and tendon are composed of varying amounts of collagen, polysaccharides, and water, and these constitu- ents mainly determine the mechanical behaviour of these tissues. ${ }^{1}$ In this respect, the muscle and tendon have elastic and viscous properties, and these can be represented as springs and damping pots respectively. ${ }^{2}$ As fig 1 shows, tendon is modelled as lying in series with the contractile elements (actin and myosin), while endomysial, perimysial, and epimysial connective tissues lie in parallel with the contractile elements. As all of these structures are viscoelastic, their resistance to lengthening is dependent on the time over which they are deformed and the rate at which the forces are applied to the tissues. ${ }^{1}$

With respect to neurological factors, stretching muscle increases the discharge of muscle spindles. ${ }^{3}$ The reflex response to a transient stretch has been shown to increase the stiffness of muscle considerably in both isolated preparations ${ }^{4}$ and in vivo muscle testing. ${ }^{5}$ The stiffness of the musculotendinous tissues will thus also be determined by the level of muscle activation. As muscle activation increases, the contractile elements stiffen, and at maximum isometric tension, contractile element stiffness can be of a similar magnitude to that of the tendon. ${ }^{6}$

The aetiology of muscle and tendon strains is thought to be multifactorial. ${ }^{7}$ Among the factors implicated in these injuries is a loss of extensibility in the soft tissues. "Warm up" activities-which typically include aerobic and stretching exercises-have been advocated as an effective means of decreasing the incidence of muscle and tendon strains, the rationale being that aerobic exercises lead to increased temperatures in the tissues and this effect enhances the extensibility of the tissue. Indeed it has been shown that a muscle preconditioned by isometric contractions requires of a musculotendinous unit. Elastic elements are represented as springs, while damping elements are represented as damping pots.

Parallel element

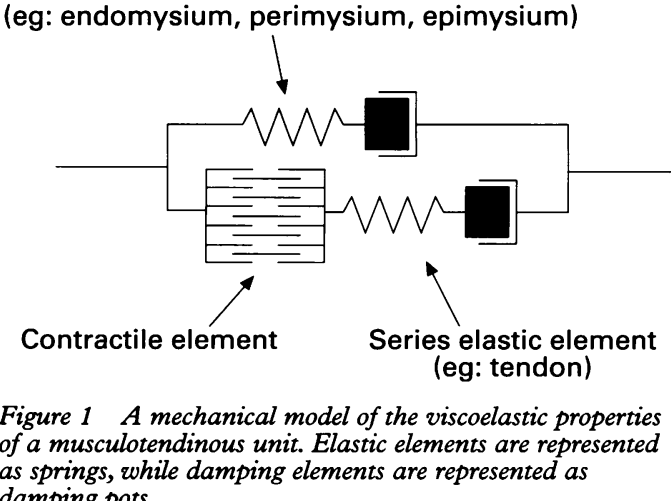


Means and standard deviations for the subjects age, height and weight

\begin{tabular}{llll}
\hline & Age (years) & Height $(\mathrm{cm})$ & Weight $(\mathrm{kg})$ \\
\hline Males & $25.0(4.5)$ & $180.0(4.5)$ & $81.0(12)$ \\
Females & $25.0(4.4)$ & $167.0(5.6)$ & $66.0(3.0)$ \\
\hline
\end{tabular}

increased force and lengthens further before rupture. ${ }^{9}$ Furthermore, there is evidence from both in vitro studies ${ }^{1011}$ and in vivo studies ${ }^{12}$ that the combination of static stretching and heat is effective in increasing the extensibility of musculotendinous structures.

To date, most in vivo studies have used changes in range of motion or displacement as the outcome measure of the effectiveness of warm up activities. ${ }^{13-15}$ In many instances, the forces associated with those changes in motion have not been quantified. Furthermore, in respect to warm up activities, no consideration has been given to the stiffness of muscle and tendon when the muscle is contracting. The current study addressed these matters in an investigation designed to determine whether passive stretching is more effective than running, or the combination of running and stretching, in producing increased range of dorsiflexion motion at the ankle joint and decreased stiffness of the plantar flexors of the ankle joint.

\section{Methods}

SUBJECTS AND EXERCISE PROTOCOLS

Twenty four subjects ( 12 male, 12 female) with no musculoskeletal problems participated in this study. The mean and standard deviation of their height, weight, and age is presented in the table. All subjects were recreational athletes who played sport for fitness (one to three times per week), but were not performing competitively in a particular sport. No subjects were engaged in a specific stretching programme, but all used stretches to warm up before sporting activities. All procedures in the study were approved by the Institute's ethics committee. Each subject undertook all of the following protocols, the order of which was randomly assigned. The stretching protocol involved five $30 \mathrm{~s}$ static stretches with $30 \mathrm{~s}$ rest between stretches. A $30 \mathrm{~s}$ duration of stretching has been shown to be effective ${ }^{15}$ in improving range of motion. The number of repetitions and the rest period were arbitrarily chosen; however, they are not atypical of what an individual on a stretching programme might undertake. The stretching technique, typically referred to as a soleus stretch, involved the subjects standing an arms length from a wall, with their hands placed on the wall for balance. The subjects stood in a step-standing position and stretched the plantar flexors by flexing both knees (fig 2). They were instructed to maintain a static hold in as flexed a position as they could maintain without pain. In the aerobic exercise protocol, subjects ran on a treadmill for 10 minutes at $60 \%$ of maximum age predicted heart rate. In the combined protocol, subjects ran first and then stretched.

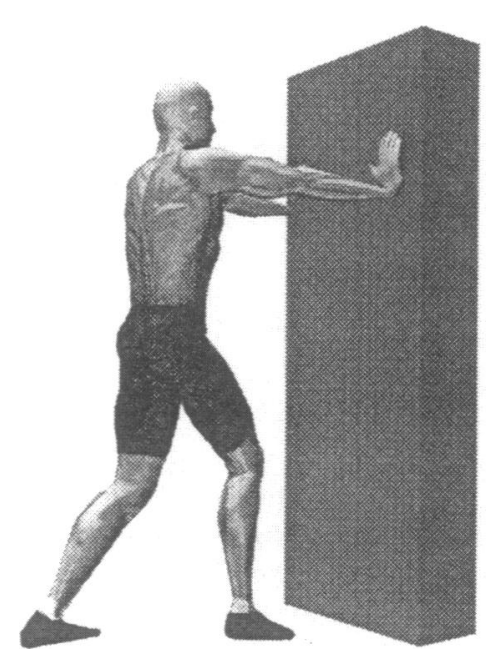

Figure $2 A$ schematic of the position in which subjects stretched their soleus muscle.

TESTING PROCEDURES

Subjects undertook the following procedures at the same time of day over a three day period. The dominant leg of subjects was used in the testing. The order of the testing procedures was randomly assigned and each test was undertaken immediately following the stretching and running protocols.

DORSIFLEXION RANGE OF MOTION MEASUREMENT OF THE ANKLE

Dorsiflexion range of motion of the ankle was assessed using weights and a pulley system as shown in fig 3 . Subjects were positioned in sitting with the knee and ankle flexed to 90 degrees and were asked to relax their lower leg muscles. The addition of weights each weighing $1.8 \mathrm{~kg}$ to the pulley system passively dorsiflexed the ankle. An electrogoniometer (Penny and Giles Ltd, Gwent, England) measured the ankle angle with each successive weight increment. Weights were added until the maximum range of dorsiflexion was attained. However, the maximum range of motion was not used in the analysis as this position was often accompanied by EMG activity from the plantar- and dorsiflexors. To avoid this activity, the position attained one weight increment before the maximum range of dorsiflexion was used in the analysis.

\section{STIFFNESS PROCEDURES}

Insofar as muscle has been shown to be a viscoelastic structure, ${ }^{16}$ it may be modelled as a mass-spring system with a damping element. The stiffness of this system can be determined by perturbing the system and examining the frequency at which it oscillates, together with the rate of decay of the oscillations (see Appendix). These variables are in turn influenced by the mass attached to the system. A schematic representation of how this system was used to measure the active stiffiness of the plantarflexor muscles is presented in fig 4. Subjects were seated with the knee and ankle flexed to 90 degrees. The lower leg, foot, and the distal aspect of the thigh are the inertial mass. A weight holder was positioned on the distal end 


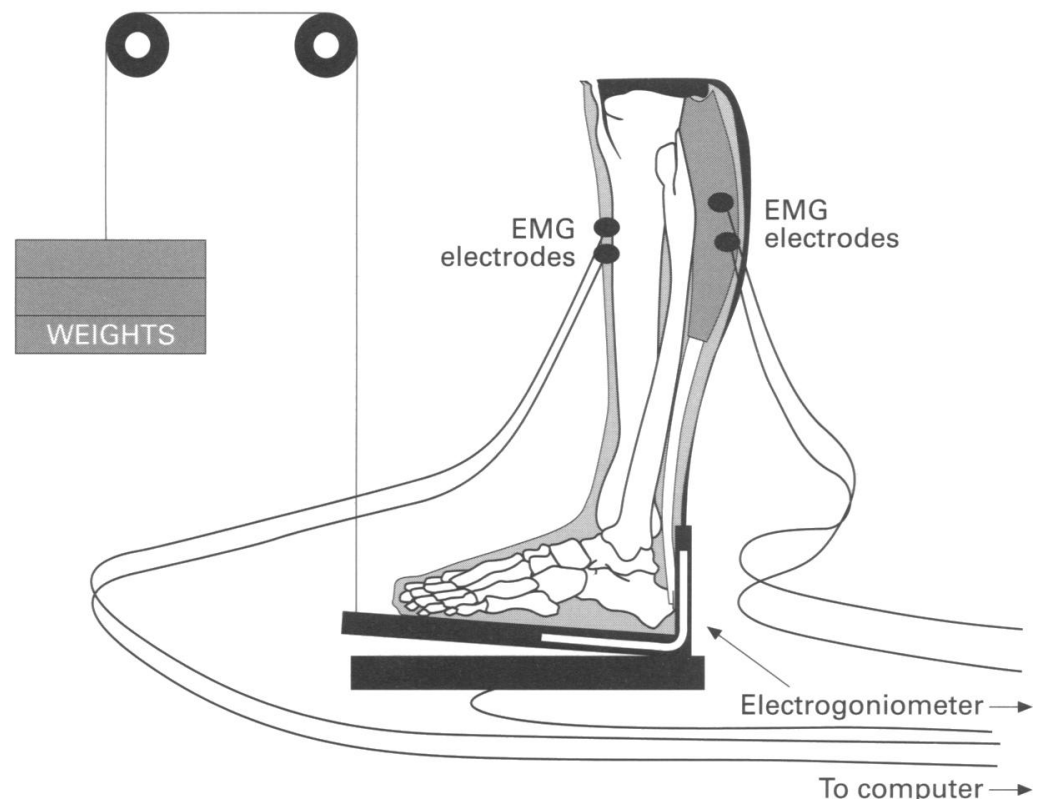

Figure $3 A$ schematic representation of the apparatus used to measure dorsiflexion range of motion of the ankle.

of the femur centred over the lower leg. One day before the subjects began the protocols (running/stretching), the maximum voluntary effort $(100 \% \mathrm{MVE})$ of the plantar flexors in the limb position described above was determined by adding weights to the weight holder resting on the distal thigh until the subject could not hold the foot parallel to the floor. Between each weight increment, one to two minutes rest was provided to prevent fatigue of the tested muscles. Having established the $100 \%$ MVE level, the weights required for $30 \%$ MVE were then calculated. This loading level was chosen as it is typical of the muscle activation level observed during gait type activities. ${ }^{17}$

A uniaxial accelerometer was secured to the weight holder. The recording axis of the accelerometer was aligned parallel to the lower leg. While loaded at $30 \%$ MVE, a brief gentle

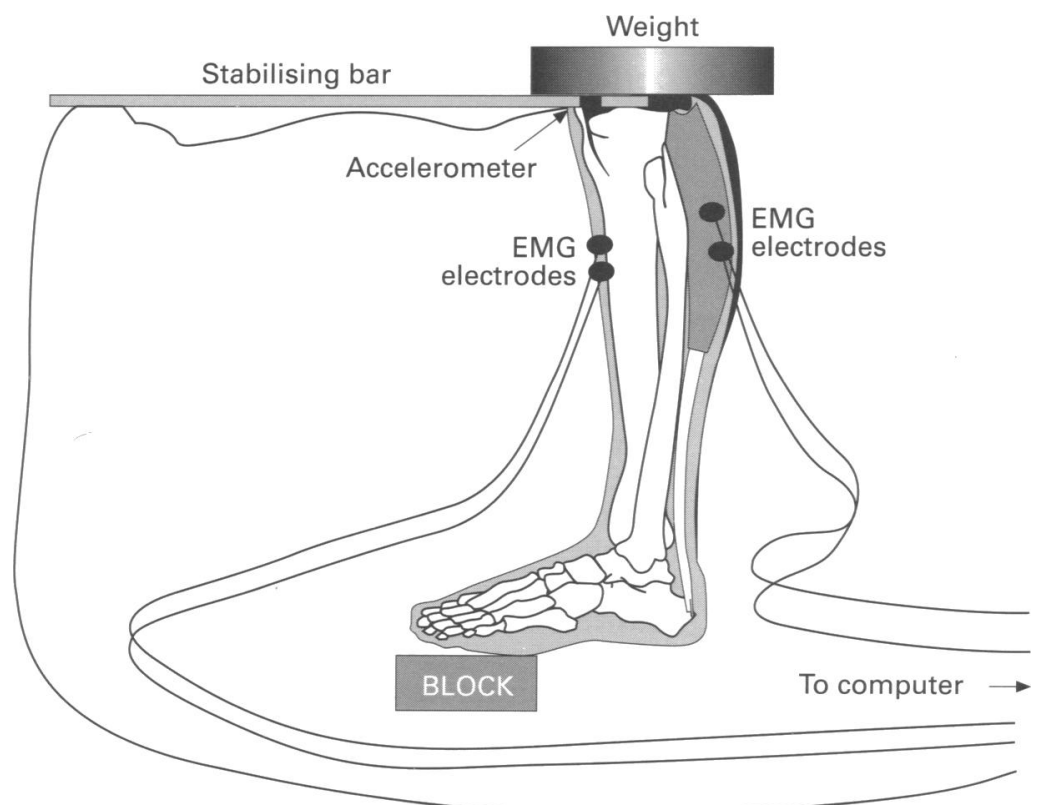

Figure 4 A schematic representation of the apparatus used for testing stiffness of the ankle plantar flexors. downward force was manually applied to the superior aspect of the weight holder. Although the magnitude of this force will not affect the frequency of oscillation, it should only be sufficient to initiate the oscillation at the ankle joint. The perturbation and resulting oscillations were detected by the accelerometer, and the signals were amplified 100 times by a strain gauge amplifier and relayed to a computerised data acquisition package at a sampling frequency of $500 \mathrm{~Hz}$. Surface electromyographic activity from the plantar and dorsiflexor muscles (3M Ag-AgCl electrodes, $5 \mathrm{~dB}$ down at 3 and $1000 \mathrm{~Hz}$ ) was monitored to ensure that subjects were not voluntarily oscillating the limb after the perturbation. EMG activity was also inspected for the presence of cocontraction. Three trials were undertaken before and after each intervention, and the means of these trials were used in the analysis of these data.

\section{STATISTICAL ANALYSIS}

The statistical analysis of these data involved a within-subjects analysis of covariance. The values obtained before each intervention served as the covariate, while the postintervention values served as the response variable. These procedures are detailed by Fleiss. ${ }^{18}$ Scheffe's tests were used for the post hoc comparisons of the stretching, running, and combination protocols. A repeated measures analysis of variance (ANOVA) of the baseline data collected each time the subject came to the laboratory was also undertaken. In all tests, the $\alpha$ level set was set to 0.05 .

\section{Results}

Data from 21 subjects were used in the statistical analysis. Two subjects failed to complete all the protocols and one subject's data were disregarded for technical reasons. No significant differences $(P>0.05)$ were observed across the baseline data for either stiffness or range of motion. The results for dorsiflexion range of motion of the ankle are presented in fig 5. There was a significant difference between the protocols $(P<0.05)$. With respect to stretching and the combined protocol, dorsiflexion range of motion increased from a mean 18.8 (SD 6.6) and 18.3 (6.2) degrees to 20.3 (6.5) and 20.6 (5.6) degrees respectively. Dorsiflexion was relatively unchanged following the running protocol [18.6 (6.6) $v 18.8$ (6.1) degrees]. Post hoc analysis indicated that the combination protocol and the stretching protocol were significantly different from the running protocol. In this respect, the percentage improvement in dorsiflexion range of motion for the combination, stretching, and running protocols were 13,8 , and 1 per cent respectively.

Figure 6 shows the results for active stiffness of the plantar flexors. In respect to the running and combined protocols, active stiffness decreased from 15034 (SD: 6500) and 15150 (SD: 6730 ) $\mathrm{N} \mathrm{m}^{-1}$ to 14104 (SD: 6404) and 14684 (SD: 6621 ) $\mathrm{N} \mathrm{m}^{-1}$ respectively. Active stiffness was relatively unchanged following stretching (cf: 15212 (SD: 6287) and 15432 


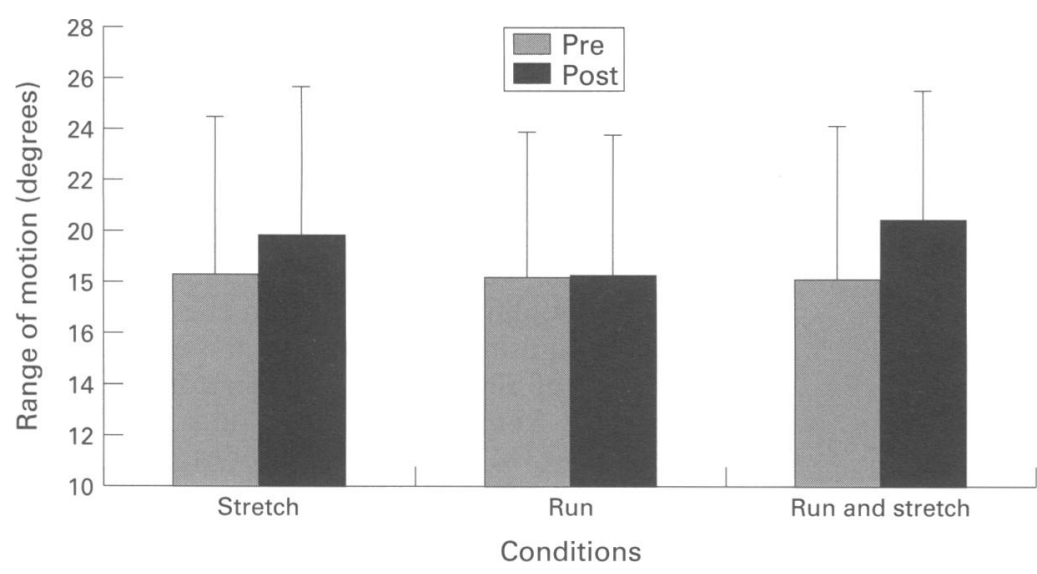

Figure 5 The results for dorsiflexion range of motion of the ankle. (Data are means and SD.)

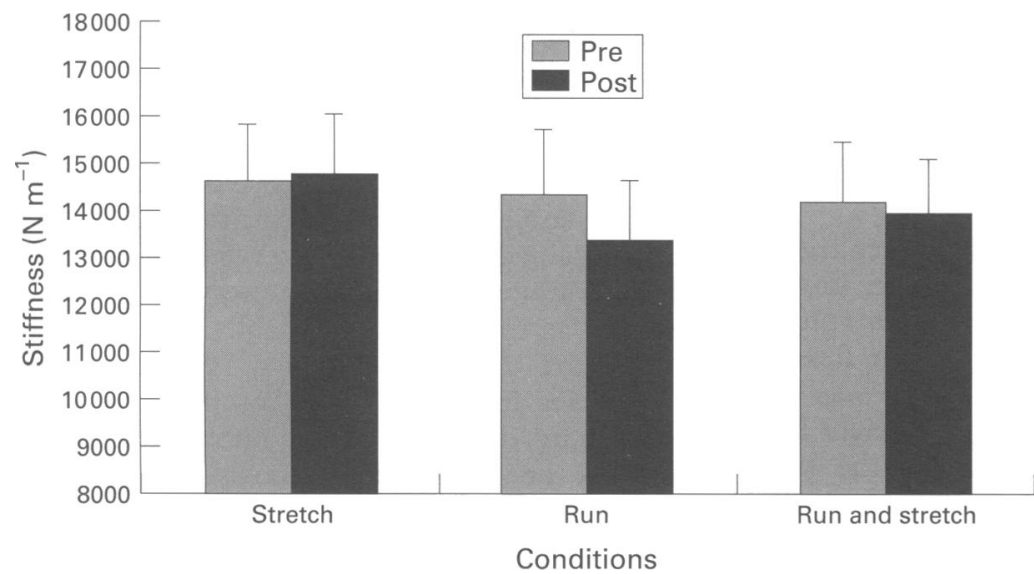

Figure 6 The results for stiffness of the ankle plantar flexors (data are means and SD).

(SD: 6868$) \mathrm{N} \mathrm{m}^{-1}$ ) Statistically, running was significantly $(P<0.05)$ more effective than stretching for decreasing muscle stiffness. The combination of running and stretching was not significantly different $(P>0.05)$ from either the running or stretching only protocols. The percentage decrease in stiffness for the running, combination and stretching protocols were 7,3 , and -1 per cent respectively.

\section{Discussion}

It has been suggested that warm up exercise can increase range of motion about a joint and also decrease the stiffness of the soft tissues. ${ }^{19}$ In doing so, the incidence of injury may be decreased. In the current study, the effects of jogging and static stretching on range of motion and stiffness were examined. The findings showed that these exercises had different effects on dorsiflexion range of motion at the ankle joint and active muscle stiffness of the plantar flexors.

Increasing range of motion is particularly important in those sports where competitors must attain extreme ranges of motion (for example, gymnastics). In the current study, to increase range of motion, the combination protocol and the stretching only protocol were significantly more effective than running only. These findings may be related to the amount of strain being placed upon the various components of the musculotendinous unit. During running, the plantar flexors are unlikely to be taken through a full range of motion at the ankle joint, and furthermore, during muscle activation the parallel connective tissue elements of the muscle are in a less tensioned length. In contrast, during passive stretching, a greater muscle length and joint range of motion is attained, perhaps placing greater tension on the capsular and parallel connective tissue structures, leading to subsequent lengthening. Although there was a trend for a greater effect when using a combined protocol $(13 \%$ increase) versus a stretching only protocol $(8 \%$ increase), no statistical difference was observed. This finding was in contrast to that of Williford $e t a l{ }^{13}$ who observed that running and stretching were more effective than stretching only. However, in the latter study the effect of the applied forces on the range of motion were not controlled.

The significance of the stiffness measures is related to resistance of the soft tissue to lengthening through the range of motion. For instance, a muscle which is relatively stiff may be more likely to be damaged in response to a sudden stretch than a more compliant muscle. In the present study the stiffness of the plantarflexor muscles was established by modelling the lower limb and foot as a single degree of freedom, freely vibrating mass spring system which included a damping element. This model has been used by other investigators to describe the stiffness of the musculature about the ankle ${ }^{20}$ and knee ${ }^{21}$ lower limb during landing from a jump, ${ }^{22}{ }^{23}$ and the stiffness of the upper limb during the bench press exercise. ${ }^{14}$ The variables associated with the observed stiffness values include the passive joint properties, the intrinsic muscle and tendon properties, and the effect of the stretch reflex. The methods used in the current study do not allow a delineation between the relative contributions of the above factors. Nevertheless, a greater decrease in stiffness was observed following the running protocol compared to the stretching protocol. This finding might also be related to the relative strain being placed on the anatomical structures while exercising. During running, the contractile elements and the tendon may be under more strain than the parallel tissue and the ankle joint capsule. In view of the findings on range of motion, perhaps stretching is more important for decreasing the stiffness of the parallel connective tissue and capsular structures.

The stiffness characteristics of muscle have been associated with both increments in performance and decreasing the likelihood of injury. With respect to the former, research indicates that the storage of elastic energy in the muscles and tendons during eccentric muscle work can augment concentric muscle work occurring immediately after eccentric muscle work, resulting in increased power and work. ${ }^{2425}$ Furthermore, Wilson and coworkers ${ }^{26}$ have commented that in events containing purely concentric work, it may be more beneficial to have a stiffer muscle group, as force production is enhanced under these conditions. With regard to the prevention of injury, intuitively a less stiff muscle would be benefi- 
cial as it can extend to a greater extent, allowing the applied forces to be absorbed over a greater range and longer time. In doing so, this "cushioning" effect may reduce the stress on the musculotendinous structures. An unresolved problem is that we do not know what the ideal levels of stiffness are for enhancing both performance and decreasing the chances of a musculotendinous injury.

\section{CONCLUSIONS}

The findings provide support for a beneficial effect of both jogging and static stretching exercises in individuals participating in sporting activities. With regard to increasing dorsiflexion range of motion at the ankle, our findings indicate that the best result is obtained by incorporating static stretching exercises, irrespective of whether jogging had been undertaken or not. However, jogging was more effective than stretching for decreasing muscle stiffness. Therefore before participating in sports involving the lower limbs, it is recommended that jogging be augmented by moving a joint at least through the range of motion that is likely to be used in subsequent activity. Finally, it may be that jogging and stretching are influential on different components of the musculotendinous unit. More evidence, probably from studies involving animals, is required to substantiate this conjecture.

We wish to thank the New Zealand Sport Science and Technology Board for funding associated with this project.

\section{Appendix}

The stiffness of the hamstring muscles can be calculated from a knowledge of the damped frequency of oscillation and the coefficient of damping. ${ }^{27}$ The equation to measure stiffness is usually written as:

$$
\mathrm{k}=4 \pi^{2} \mathrm{mf}^{2}+\mathrm{c}^{2} / 4 \mathrm{~m}
$$

where $k$ is the stiffness $\left(\mathrm{N} \mathrm{m}^{-1}\right), \mathrm{m}$ is the mass, $\mathrm{f}$ is the damped frequency of oscillation, and $\mathrm{c}$ is the coefficient of damping.

The coefficient of damping is obtained using a logarithmic decrement technique. Figure Al shows an oscillation decaying exponentially with time.

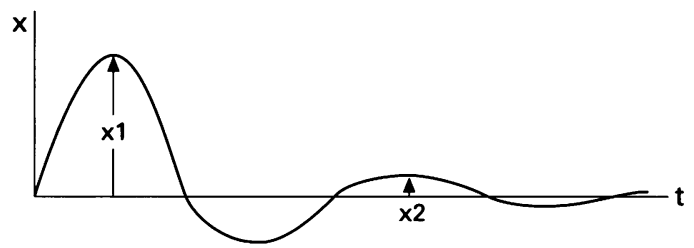

Figure A1 Graph of displacement versus time for a damped oscillation.

The amount of damping is obtained from the amplitude change in the oscillation during one complete cycle of oscillation. This may be expressed as:

$$
\delta \ln \frac{x_{1}}{x_{2}}
$$

where $\delta$ is the logarithmic decrement. The damping factor $\zeta$ may then be calculated:

$$
\zeta=\frac{\delta}{\sqrt{(2 \pi)^{2}+\delta^{2}}}
$$

The natural frequency $\left(\omega_{n}\right)$ is calculated with a knowledge of the damping factor $(\zeta)$ and the damped frequency $\left(\omega_{\mathrm{d}}\right)$ of oscillation:

$$
\omega=\frac{\omega_{d}}{\sqrt{1-\zeta^{2}}}
$$

The coefficient of damping $\mathrm{c}$ is calculated from a knowledge of the natural frequency of oscillation $\left(\omega_{n}\right)$, damping factor $(\zeta)$ and the mass $(\mathrm{m})$ :

$$
c=2 m \zeta \omega_{n}
$$

1 McNeil-Alexander R. Animal mechanics, 2nd ed. Oxford: Blackwell Scientific, 1983:67-112.

2 Enoka R. Neuromechanical basis of kinesiology. Champaign: Human Kinetics, 1988:200-6.

3 Carew TJ. The control of reflex action. In: Kandel ER, Schwartz JH, eds. Principles of neural science, 2nd ed. New Ychwartz JH, eds. Principles of neural

4 Nichols TR, Houk JC. The improvement in linearity and the regulation of stiffness that results from the actions of the regulation of stiffness that results from the action
the stretch reflex. $\mathcal{F}$ Physiol (Lond) 1976;39:119-42.

5 Sinkjaer T, Toft E, Andreassen S, Hornemann BC. Muscle stiffness in human dorsiflexors. $f$ Neurophysiol 1988;60 1111-21.

6 Morgan DL. Separation of active and passive component of short range stiffness of muscle. Am $\mathcal{F}$ Physiol 1977;232 $45-9$.

7 Kvist M. Achilles tendon injuries in athletes. Sports Med 1994;18:173-201.

8 Ekstrand J, Gillqvist J. The frequency of muscle tightness and injuries in soccer players. Am $\mathcal{F}$ Sports Med 1982;10: $75-8$.

9 Safran M, Garrett W, Seaber A, Glisson R, Ribbeck B. The role of warmup in muscular injury prevention. Am $\mathcal{f}$ Sports Med 1988;16:123-9.

10 Warren C, Lehmann J, Koblanski J. Heat and stretch procedures: an evaluation using rat tail tendons. Arch Phys Med Rehab 1976;57:122-6.

11 Lehmann J, Masock A, Warren C, Koblanski J. Effect of therapeutic temperatures on tendon extensibility. Arch Phys Med Rehab 1970;51:481-5.

12 Lentell G, Hetherington T, Eagan J, Morgan M. The use of thermal agents to influence the effectiveness of a low load prolonged stretch. $\mathcal{F}$ Orthop Sports Phys Ther 1992;16:200-7.

13 Williford H, East J, Smith F, Burry L. Evaluation of warm-up for improvement in flexibility. Am 7 Sports Med 1986;14:316-9.

14 Wilson G, Wood GA, Elliott BC. The relationship between stiffness of the musculature and static flexibility: An stiffness of the musculature and static flexibility: An injury. Int $\mathcal{Y}$ Sports Med 1991;12:403-7.

15 Bandy W, Irion J. The effect of time on static stretch on the flexibility of the hamstring muscles. Phys Ther 1994;74: 845-52.

16 Woledge RC. The thermoelastic effect of change of tension in active muscle. F Physiol (Lond) 1961;155:187-208.

17 Ciccoti M, Kerlan R, Perry J, Pink M. An electromyographic analysis of the knee during functional activities. $A m \mathcal{F}$ Sport Med 1994;22:645-50.

18 Fleiss J. The design and analysis of clinical experiments. New York: John Wiley and Sons, 1986:263-90.

19 Taylor DC, Dalton JD, Seaber AV, Garrett WE. Viscoelastic properties of muscle-tendon units. Am F Sports Med 1990; 18:300-9.

20 Shorten M. Muscle elasticity and human performance. Med Sci Sports Exerc 1987;25:1-18.

21 McNair PJ, Wood, GA, Marshall RN. Stiffness of the hamstring muscles and its relationship to function in anterior cruciate

22 Cavagna GA. Elastic bounce of the body. I Appl Physiol 1970;29:279-82.

23 Bach T, Chapman AE, Calvert TW. Mechanical resonance of the human body during voluntary oscillations about the ankle joint. F Biomech 1983;16:85-90.

24 Cavagna G, Dusman B, Magaria R. Positive work done by a previously stretched muscle. F Appl Physiol 1968;24:21-32.

25 Asmussen E, Bonde-Peterson F. Storage of elastic energy in skeletal muscles in man. Acta Physiol Scand 1974;91:38592.

26 Wilson GJ, Wood GA, Elliott BC. Optimal stiffness of the series elastic component in a stretch shorten cycle activity. f Appl Physiol 1991;70:825-33.

27 Thompson WT. Theory of vibrations with applications, 2nd ed. London: George Allen and Unwin; 1981:1-30. 